\title{
Is erythrocyte sedimentation rate a useful inflammatory marker independently of the hematocrit? Comparison results with plasma viscosity
}

\author{
Óscar Fuster ${ }^{\mathrm{a}}$, Amparo Vayáa,*, Cristina Giménez ${ }^{\mathrm{a}}$, José Todolíb, José L. Hernández ${ }^{\mathrm{a}}$ \\ and Begoña Laiz ${ }^{\mathrm{a}}$ \\ ${ }^{a}$ Hematology and Hemorheology Unit, Department of Clinical Pathology, Hospital Universitari \\ i Politécnic La Fe, Valencia, Spain \\ ${ }^{\mathrm{b}}$ Internal Medicine Department, Hospital Universitari i Politécnic La Fe Valencia, Spain
}

To the Editor,

Plasma viscosity (PV) and erythrocyte sedimentation rate (ESR) are tests which can be used as inflammation and tissue injury markers $[2,11]$. ESR usage is more extended than PV in clinical laboratories. However ESR may be haematocrit (Ht) dependent and is related with red blood cell (RBC) rheological properties [1], whereas RBC are not involved in PV. We aimed to evaluate retrospectively the impact of $\mathrm{Ht}$ on ESR as an inflammatory marker and compare the results with those of PV in 333 out- and in-patients at our hospital. Our study is a human non-interventional retrospective review and written consent is not required for human non-interventional studies.

We evaluated both markers in two patient groups of i.e., the first one did not present other inflammatory components measured as C-reactive protein (CRP) below $10 \mathrm{mg} / \mathrm{L}$ and fibrinogen below $450 \mathrm{mg} / \mathrm{dL}$ $(n=211)$. The second one presented CRP over $10 \mathrm{mg} / \mathrm{L}$ and/or fibrinogen values higher than $450 \mathrm{mg} / \mathrm{dL}$ $(n=122)$.

$\mathrm{PV}$ and ESR were determined in a capillary plasma viscosimeter (Fresenius $\mathrm{GmbH}$, Germany) at $37^{\circ} \mathrm{C}$ and using a Ves matic 30 Plus Analyzer (Diesse Diagnostica Senese, Siena, Italy), respectively. CRP was determined by immunoturbidity in an AU5430 autoanalyzer (Beckman Coulter, Lexington, KY, USA) and fibrinogen analysis was performed in an ACL-TOP 700 autoanalyzer (Instrumentation Laboratory, Bedford, MA, USA).

\footnotetext{
${ }^{*}$ Corresponding author: Amparo Vayá, Hemorheology and Hemostasis Unit, Service of Clinical Pathology, La Fe University Hospital, Av Campanar 21, 46009 Valencia, Spain. Tel.: +34 963862714; E-mail: vaya_amp@gva.es.
} 
Our results showed that ESR correlated negatively with the Ht in both patient groups (Non Inflammatory group: rho: $-0.528, p<0.001$ and Inflammatory group: rho: $-0.727, p<0.001)$. As it was be expected, PV did not correlate with Ht because it do not contain RBC (Non Inflammatory group: rho: -0.079 , $p=0.255$ and Inflammatory group: rho: $-0.099, p=0.276$ ).

Moreover, we studied ESR and PV in both patient groups according to the different hematocrit values (below 36\%, between 36-45\% and higher than 45\%). We found significant diferences in ESR between the Ht groups ( $p<0.001$ in both groups), but not in PV ( $p=0.480$ in the non-inflammatory group and $p=0.161$ in the inflammatory group) (Fig. 1).

Finally, we studied the probability of PV and ESR discriminating both inflammatory states (CRP below $10 \mathrm{mg} / \mathrm{L}$ and fibrinogen under $450 \mathrm{mg} / \mathrm{dL}$ vs. CRP over $10 \mathrm{mg} / \mathrm{L}$ and/or fibrinogen values over $450 \mathrm{mg} / \mathrm{dL}$ ) across the three hematocrit patient groups. The PV cut-off value was $1.33 \mathrm{mPa} . \mathrm{s}$, and the ESR cut-off points were $30 \mathrm{~mm} / \mathrm{h}$ for women and $20 \mathrm{~mm} / \mathrm{h}$ for men. Our results showed that $\mathrm{PV}$ has a higher predictive negative value (PNV) and predictive positive value (PPV) than ESR to discriminate the inflammatory state in patients with $\mathrm{Ht}$ below $36 \%$ or higher than $45 \%$ (Table 1).

Our results suggest that ESR may be influenced by Ht unlike PV, which may be useful as an inflammatory marker independently of the Ht values. Different authors have suggested that ESR is dependent of red blood cell factors, such as $\mathrm{Ht}$, red cell aggregability and deformability and influenced by demographic data $[8,10]$. Even others authors have suggested that ESR should be replaced with PV because of its

Non Inflammatory patients
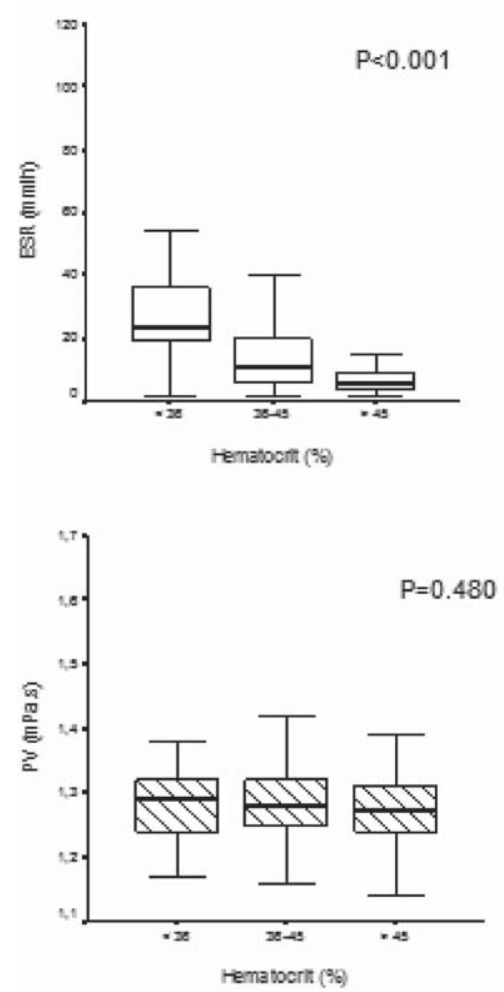

Inflammatory patients
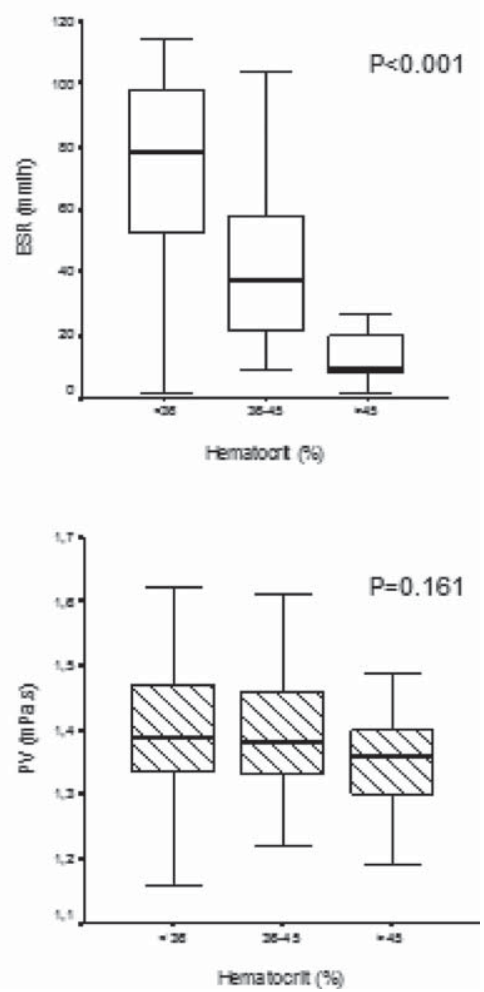

Fig. 1. Erythrocyte sedimentation rate and plasma viscosity in patients with positive and negative inflammatory markers according to the hematocrit. 
Table 1

Predictive negative and predictive positive values of erythrocyte sedimentation rate and plasma viscosity to discriminate inflammatory states

\begin{tabular}{lcc}
\hline & PNV & PPV \\
& $\%($ CI 95\%) & $\%($ CI 95\%) \\
\hline Hematocrit $<36 \%(n=41)$ & & \\
PV & $84(65-100)$ & $61(31-91)$ \\
ESR & $81(54-100)$ & $45(22-68)$ \\
Hematocrit 36-45\% $(n=160)$ & & \\
PV & $89(82-96)$ & $44(26-62)$ \\
ESR & $92(86-98)$ & $47(31-63)$ \\
Hematocrit $>45 \%(n=132)$ & & \\
$\quad$ PV & $89(81-96)$ & $43(21-65)$ \\
ESR & $82(74-90)$ & $33(2-64)$ \\
\hline
\end{tabular}

independence of red cell changes, slight variation with demographic factors and proven measure of disease activity [4-8]. Although PV offers advantages over ESR, different studies have generally shown a reasonable correlation between PV and ESR in a variety of organic diseases, such as rheumatic disease, chronic infections, and malingnancy [5, 9]. However our results show that PV has greater discrimination power for the inflammatory state than ESR in patients with $\mathrm{Ht}$ under $36 \%$ or over $45 \%$ in the studied population. Despite our results, PV and ESR could display a different behaviour in different clinical settings. So, there are discrepancies in the choice of PV or ESR in several pathologies where ESR has been traditionally used such as rheumatic disorders or temporal arteritis [4, 6, 9]. In this sense the election of one of both tests could be conditioned by the presence of ESR influencing factors. Therefore, further studies on specific diseases that take into account the factors that can modify both parameters are required. In any case, the two tests can be used together to improve PNV and PPV.

In summary, our results imply that PV is an inflammatory marker that is independent of the $\mathrm{Ht}$ in contrast to ESR, which is mainly related with the $\mathrm{Ht}$ and RBC rheological behaviour. The usefulness of ESR should be reviewed in pathologies which imply reductions or increases in the Ht.

\section{References}

[1] A. Cortinovis, A. Crippa, M. Crippa, T. Bosoni and R. Moratti, Relations between plasma-erythocyte viscosity factors and ESR, Minerva Cardioangiol 40 (1992), 323-330.

[2] G.J. Dinant, J.W. van Wersch and H.S. Goei The and J.A. Knottnerus, Plasma viscosity and erythrocyte sedimentation rate in inflammatory and non-inflammatory rheumatic disorders, Clin Rheumatol 11 (1992), 66-71.

[3] M. Gudmundsson and A. Bjelle, Plasma viscosity in the monitoring of therapy in rheumatoid arthritis patients, Scand $J$ Rheumatol 24 (1995), 219-224.

[4] E.J. Kanfer and B.A. Nicol, Haemoglobin concentration and erythrocyte sedimentation rate in primary care patients, $J R$ Soc Med 90 (1997), 16-18.

[5] G.D. Lowe, Should plasma viscosity replace the ESR? Br J Haematol 86 (1994), 6-11.

[6] G. Késmárky, P. Kenyeres, M. Rábai and K. Tóth, Plasma viscosity: A forgotten variable, Clin Hemorheol Microcirc 39 (2008), 243-246 
[6a] A. Manfredi, M. Sebastiani, V. Carraro, M. Iudici, M. Bocci, G. Vukatana, R. Gerli, R. De Angelis, P. Del Medico, E. Praino, A. Lo Monaco, R. D'Amico, C. Del Giovane, S. Mazzuca, M. Colaci, D. Giuggioli, C. Ferri, Prediction risk chart for scleroderma digital ulcers: A composite predictive model based on capillaroscopic, demographic and clinico-serological parameters, Clin Hemorheol Microcirc (2014). DOI: 10.3233/CH-141809

[7] P.T. Murphy, B. Allen and R.M. Hutchinson, Plasma viscosity and erythrocyte sedimentation rate in suspected cases of temporal arteritis, Br J Haematol 87 (1994), 671.

[8] T. Ng, Erythrocyte sedimentation rate, plasma viscosity and C-reactive protein in clinical practice, Br J Hosp Med $\mathbf{5 8}$ (1997), 521-523.

[9] H.E. Paulus, B. Ramos, W.K. Wong, et al., Equivalence of the acute phase reactants C-reactive protein, plasma viscosity, and Westergren erythrocyte sedimentation rate when used to calculate American College of Rheumatology 20\% improvement criteria or the Disease Activity Score in patients with early rheumatoid arthritis. Western Consortium of Practicing Rheumatologists, J Rheumatol 26 (1999), 2324-2331.

[10] E. Piva, M.C. Sanzari, G. Servidio and M. Plebani, Length of sedimentation reaction in undiluted blood (erythrocyte sedimentation rate): Variations with sex and age and reference limits, Clin Chem Lab Med 39 (2001), 451-454.

[11] A. Manfredi, M. Sebastiani, V. Carraro, M. Iudici, M. Bocci, G. Vukatana, R. Gerli, R. De Angelis, P. Del Medico, E. Praino, A. Lo Monaco, R. D’Amico, C. Del Giovane, S. Mazzuca, M. Colaci, D. Giuggioli, C. Ferri, Prediction risk chart for scleroderma digital ulcers: A composite predictive model based on capillaroscopic, demographic and clinico-serological parameters, Clin Hemorheol Microcirc (2014). DOI: 10.3233/CH-141809 